\title{
Increased gaze following for fearful faces. It depends on what you're looking for!
}

\author{
Gustav Kuhn • Jason Tipples
}

Published online: 16 November 2010

(C) Psychonomic Society, Inc. 2010

\begin{abstract}
An oculomotor visual search task was used to investigate how participants follow the gaze of a nonpredictive and task irrelevant distractor gaze, and the way in which this gaze following is influenced by the emotional expression (fearful vs. happy) as well as participants' goal. Previous research has suggested that fearful emotions should result in stronger cueing effects than happy faces. Our results demonstrated that the degree to which the emotional expression influenced this gaze following varied as a function of the search target. When searching for a threatening target, participants were more likely to look in the direction of eye gaze on a fearful compared to a happy face. However, when searching for a pleasant target, this stronger cueing effect for fearful faces disappeared. Therefore, gaze following is influenced by contextual factors such as the emotional expression, as well as the participant's goal.
\end{abstract}

Keywords Gaze cueing · Gaze following · Visual Search . Emotion $\cdot$ Attentional control setting

\section{Introduction}

Other peoples' eyes provide us with much valuable information. For example, where someone looks often reveals her interest and the locus of her attention. Things

\footnotetext{
G. Kuhn $(\bowtie)$

Department of Psychology, Brunel University,

Uxbridge, Middlesex UB8 3PH, UK

e-mail: gustav.kuhn@brunel.ac.uk

J. Tipples

Department of Psychology, The University of Hull,

Hull HU6 7RX, UK

e-mail: J.Tipples@hull.ac.uk
}

that are of interest to us are often of interest to others, and it may therefore come as no surprise that we often attend to where others look. Indeed, observing the gaze direction of another person has a powerful effect on our own attention attentional orienting in response to eye gaze appears to be automatic and obligatory (Driver, Davies, Kidd, Maxwell, Ricciardelli, \& Baron-cohen, 1999; Friesen, Ristic, \& Kingstone, 2004) (cf. Vecera \& Rizzo, 2006). It has been suggested that attentional orienting in response to eye gaze may be of evolutionary advantage, as it may facilitate the detection of potential dangers or threats. Directional eye gaze is only one of several cues that may indicate the presence of a danger. For example, the emotional expression of a face provides information about the motivational state of a person, and as such may provide cues for potential danger (Ekman \& Oster, 1979). By combining the emotional and the directional facial information, we can discover the existence as well as the location of a potential threat. For example, a person with a fearful expression with eyes averted may indicate to the observer "Watch Out! There is potential danger at this location". In short, it seems reasonable to predict that specific emotional expressions will modulate attentional orienting in response to eye gaze.

Attentional orienting in response to eye gaze is typically investigated using a Posner type gaze cueing paradigm (Friesen \& Kingstone, 1998). In the gaze cueing task, participants are asked to identify targets appearing to either side of a face looking either to the left or the right. Numerous studies have shown that targets appearing at the looked at location (valid) are detected more rapidly than those appearing on the opposite side (invalid), even when the gaze direction is non-predictive or counter-predictive of the upcoming target location (Frischen, Bayliss, \& Tipper, 2007; Langton, Watt, \& Bruce, 2000). Recent eye movement studies in which participants were asked to watch 
video clips in which the gaze direction of a magician was experimentally manipulated have shown that people often look at locations that are being looked at by others (Kuhn \& Land, 2006; Kuhn, Tatler, \& Cole, 2009). These studies demonstrate that under free viewing conditions gaze cues play an important role in determining where we look. Moreover, more experimentally controlled studies have shown that participants are more likely to execute saccades in the direction indicated by the distractor eyes (Kuhn \& Benson, 2007; Kuhn, et al., 2010; Kuhn \& Kingstone, 2009; Mansfield, Farroni, \& Johnson, 2003; Ricciardelli, Bricolo, Aglioti, \& Chelazzi, 2002), even when doing so is detrimental to the task (Kuhn \& Kingstone, 2009), leading to the conclusion that gaze following is an automatic process.

Research into the modulation of gaze cueing by the emotional expression has not always recorded an effect of expression on attentional orienting. In a series of six experiments, Hietanen and Leppanen (2003) consistently demonstrated that gaze cueing was independent of the emotional expression (happy, neutral, fearful, angry). However, the effects are more consistent for individuals who report high levels of emotion and also, when stimulus displays involve dynamic, multi-frame transformations of expressions rather than a single, static display of a person expressing an emotion. For example, individuals with high levels of self-reported trait anxiety show stronger gaze cueing in response to fearful than happy faces (Mathews, Fox, Yiend, \& Calder, 2003). Similarly, Tipples (2006) found stronger gaze cueing effects for fearful faces in high compared to low fearful participants. Two studies (Fichtenholtz, Hopfinger, Graham, Detwiler, \& Labar, 2007; Putman, Hermans, \& Van Honk, 2006) have recorded enhanced gaze cueing by fearful expressions in unselected participants when dynamic expression changes were used. In short, research suggests that gaze cueing does appear to be influenced by the facial expression but the degree to which this occurs may depend on both the individual (i.e., anxiety level) as well as the exact presentation of the gaze cue (i.e., dynamic vs. static).

When monitoring for threats and dangers it makes intuitive sense to prioritize fearful faces, and thus we would expect a stronger gaze cuing effect for fearful emotions. However, there are circumstances when other expressions might produce stronger gaze cueing effects. For example, when searching for a reward, happy faces may be more informative than fearful faces, and it would make sense for us to prioritize the former. In the visual search literature there is evidence to suggest that the extent to which a stimulus feature captures our attention depends on the search goal (Folk, Remington, \& Johnston, 1992). For example, you are more likely to be distracted by red items if you are searching for something that is red, than when you are searching for a different colored item (Irwin, Colcombe, Kramer, \& Hahn, 2000). These studies illustrate that most attentional processes are modulated by top down control, and as such, a reasonable suggestion is that gaze cueing will be enhanced when a facial expression is informative with respect to the search task. The aim of the current experiment was to investigate whether the participant's search goal will predict the way in which gaze cueing is influenced by emotional expression. In particular, when the task is to search for threat rather than to search for a pleasant target, we predict a stronger cueing effect for fearful than for happy faces. Conversely, when the task is to search for a pleasant target we predict stronger cueing for happy facial expressions.

Rather than using a typical gaze cueing paradigm, we developed a novel gaze cueing task, which was thought to represent the everyday experience of using social attention more closely. Participants performed a visual search task in which they were required to search for a target item amongst several distractor items, whilst having their eye movements monitored using an eye tracker. Independent of the search display, the eye gaze from a centrally presented face cued the location of one of the target locations. As the gaze direction was determined randomly, and was nonpredictive of the target location, systematic biases in eye movement behavior in response to this distractor gaze are indicative of eye movements being influenced by the distractor eyes. This novel design allows us to manipulate participants' search goal independently of the search display.

\section{Method}

\section{Subjects}

Twenty-two Durham University Undergraduate students participated for course credits (15 female). All participants had normal or corrected-to-normal vision, and were naïve to the purpose of the experiment. Mean age was 25.4 years $(\mathrm{SD}=7.36)$.

\section{Stimuli}

A short description of each stimulus is provided in the Supplementary Materials. There were 24 different sets of images (each containing four animal pictures). Nine participants (five male, four female; mean age $=32$ ) rated the stimuli for pleasantness and threat. Specifically, participants were asked to indicate the extent to which they found the pictures pleasant (where $1=$ very unpleasant, $4=$ neither pleasant nor unpleasant, $7=$ very pleasant) and threatening (using a 7-point scale where $1=$ not at all and 
7 = very threatening). The pictures were presented in a new random order for each participant. A one-way repeated measures ANOVA was conducted on the pleasantness and threat ratings. The ANOVA was significant for both the pleasantness $(\mathrm{F}(2,16)=106.12, p<0.001)$ and threat ratings $(\mathrm{F}(2,16)=91.67, p<0.001)$. Multiple $t$ tests (using the Bonferroni correction, $p=0.01$ ) were used to examine the effect of stimulus emotion (pleasant, threat, neutral). Ratings of pleasantness were higher for the pleasant pictures compared to both the neutral and threatening pictures and lower, for the threatening pictures compared to both the neutral and pleasant pictures. Ratings of threat were higher for the threatening pictures compared to both the neutral and pleasant images. The neutral and pleasant images did not differ with respect to threat ratings.

\section{Material and procedure}

Eye movements were monitored monocularly at $500 \mathrm{~Hz}$, using an Eyelink II (SR Research Ltd, Osgoode, Canada). The experiment was run on a Pentium D computer and displayed on a 20-in CRT monitor $(1024 \times 768 ; 85 \mathrm{~Hz})$. Viewing distance was $63 \mathrm{~cm}$. The experiment was compiled and run using Experiment Builder (SR Research Ltd, Osgoode, Canada). Each trial began with a central white fixation point $\left(0.63^{\circ}\right.$ in diameter $)$ on a black background (see Fig. 1). Participants were asked to fixate on this central spot and press the space bar once they had achieved fixation. This procedure allowed us to perform a drift correction and ensured that participants fixated the center of the screen. The keypress initiated the remaining trial sequence. Each trial started with a neutral face looking ahead. The faces were created using Poser 5.0 ${ }^{\circledR}$ (Curious Labs Inc., Santa Cruz, CA). After 1,500 ms, the face started to look either to the left, right, up or down and the emotional expression changed to fearful or happy. In order to ensure that the change in emotion and gaze direction appeared as natural as possible, morphed sequences of faces were used and the shift in eye direction occurred gradually over a sequence of ten steps, which lasted for $120 \mathrm{~ms}$. Moreover, special care was taken to ensure that the eyes of the fearful and the happy faces were identical (same pupil and sclera), as well as the movement component of the eyes (e.g., pupils, eye lids). Three hundred milliseconds after the dynamic sequence was completed images of four animal pictures measuring $5.4^{\circ} \times 5.4^{\circ}$ (gray scale) appeared equidistant from fixation $\left(5.2^{\circ}\right)$ left, right, up or down from fixation. The animal pictures were previously rated by ten participants in terms of how pleasant / threatening they were perceived to be. Each trial contained one threatening one pleasant and two neutral animals. The threatening and pleasant animals were equally likely to appear in any of the four locations. For each of the animal sets the central face looked in each of the four directions, and changed to each of the two emotions (fearful and happy) resulting in 192 trials in total [24(images) $\times 4$ (direction) $\times 2$ (emotion)]. Participants were asked to fixate the fixation point and urged not to move their eyes until the search display appeared. Depending on the task, participants were required to search for the most threatening/pleasant animal in the display as quickly as possible. Once they have found it, they were required to fixate the selected animal and press the space bar to indicate their selection. Participants were instructed to ignore the central face and were told that the emotional expression as well as the gaze direction of this central face was just as likely to look in either of the four directions (i.e., up, down left right), and was just likely to change into a fearful or a happy emotional expression. Moreover, participants were informed that the target locations were determined randomly and thus independent of the gaze direction or emotional expression.

Participants were presented with a block of 192 trials on which they were asked to search for the most threatening animal, and a block of 192 trials in which they were required to search for the most pleasant target. The order of blocks was balanced across subjects.

\section{Results}

Eye movements were analyzed from the point at which the search display was presented, and were classified according to their direction (left, right, up, down). Figure 2 shows the percentage of trials on which the first saccade was made in the same direction in which the distractor eyes were looking. Only saccades that were executed after the search display appeared were analyzed. The first saccade was defined as eye movements with velocities and accelerations exceeding $30 \% \mathrm{~s}$, and $8,000 \% \mathrm{~s}^{2}$, and exceeding $1^{\circ}$ in amplitude. Trials on which the first saccade went in the same direction in which the eyes were looking were classified as gaze following trials. As the gaze direction was not predictive of the search target, and there were four potential saccade targets, gaze following on more than $25 \%$ of the trials indicates an above chance gaze following. From the 95\% confidence intervals in Fig. 2 it is clear that participants were significantly more likely to saccade in the cued, rather than any of the other directions, thus illustrating a strong tendency for gaze following.

Our main interest was whether the level of gaze following was influenced by the emotional expression, and whether this varied as a function of search target. An ANOVA with search target (pleasant target vs. threatening target) and emotion (fearful vs. happy) on the percentage of gaze following trials, found no significant main effect of search target $\mathrm{F}(1,21)=0.078, p=0.78$, and no significant main effect of emotion $\mathrm{F}(1,21)=1.15, p=0.23$. However, there 


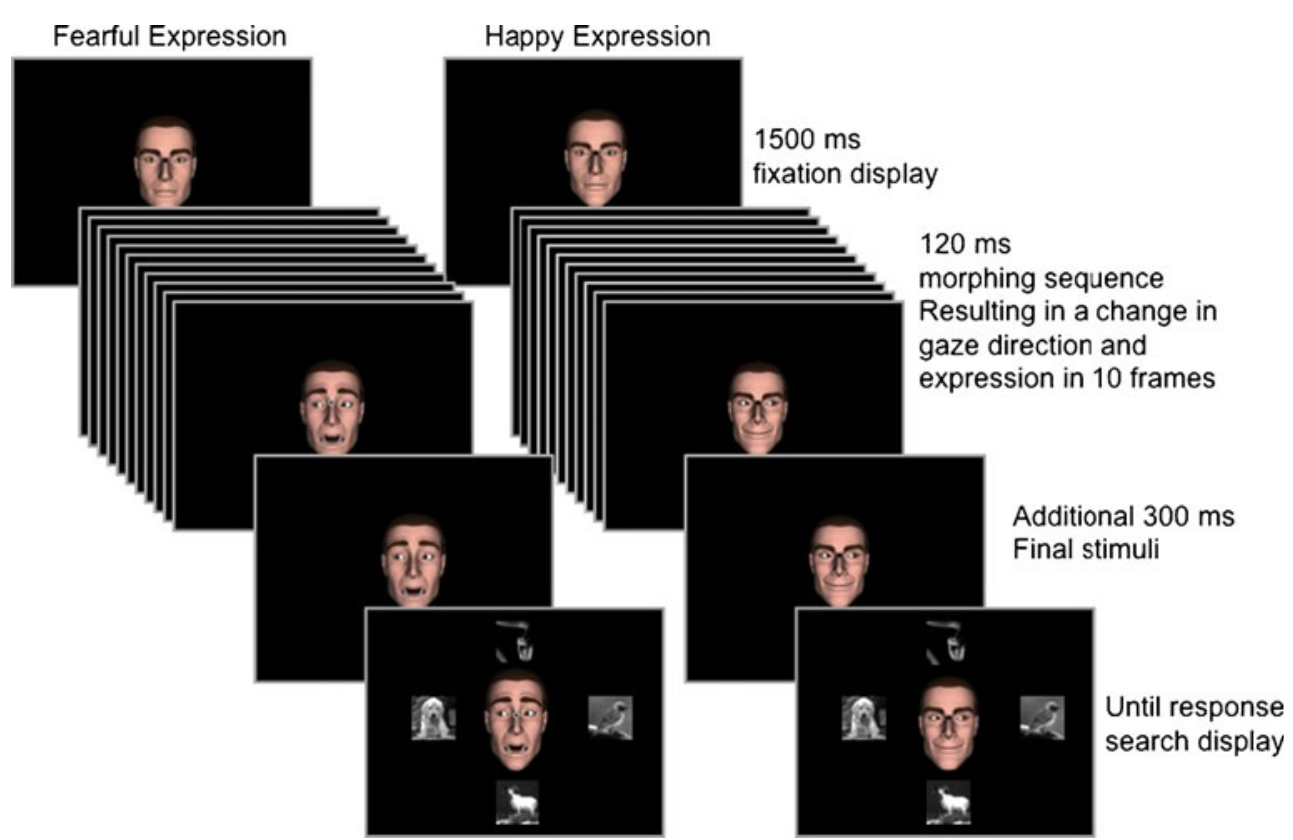

Fig. 1 The order of events for one of the fearful and the happy emotional expression trials. After the fixation display, a $120-\mathrm{ms}$ dynamic stimuli sequence (ten frames) displaying a face gradually increasing its facial expression from neutral to either fearful or happy, and the eyes simultaneously gradually looking to the right, left up or down. The final frame was displayed for $300 \mathrm{~ms}$, after which the search display consisting of four animal pictures was presented. One

was a significant emotion by search target interaction $\mathrm{F}(1,21)=10.7, p=0.004$. When searching for a threatening target, there was significantly more gaze following when presented with a fearful than for a happy face $\mathrm{t}(21)=2.93$, $p=.008$ (see Fig. 2). In contrast, when searching for a pleasant target the degree of gaze following for fearful faces was numerically, but not significantly lower than for happy faces $\mathrm{t}(21)=-1.55, p=0.34$ (see Fig. 2). ${ }^{1}$

In addition to looking at the direction in which saccades are executed, gaze cues also affect the speed at which saccades are initiated (Kuhn \& Benson, 2007). We therefore measured the time it took for participants to initiate a saccade, depending on whether this saccade was congruent to the distractor eyes or it went in another direction. Saccade onset latency was defined as the time to initiate a saccade after the search display appeared. Saccade latencies shorter than $80 \mathrm{~ms}$ were classified as outliers and excluded from the analysis. ${ }^{2}$ These outliers accounted for

\footnotetext{
$\overline{{ }^{0} \text { We conducted }}$ an additional analysis to see whether gaze following as a function of whether the first saccade went towards the correct or incorrect target. Saccades were executed more frequently towards the target than any of the other animal pictures $\mathrm{F}(1,21)=10.5, p=0.004$. However, gaze following was independent of the whether the correct or incorrect target was fixated $(p>0.05)$

${ }^{0}$ Data from all trials was included regardless of whether the saccade whet towards the correct or incorrect target.
}

of these was always threatening, one pleasant, and the other two were neutral. In the pleasant search target condition, participants were required to search for the most pleasant animal and press the space bar while fixating it, to indicate the selection. In the threatening search target condition, participants were required to search for the most threatening animal. The figure is not drawn to scale

$10.9 \%(\mathrm{SD}=9.98)$ of all trials. Closer analysis of these outliers revealed even though the chance of the target appearing at the gazed at location was only $25 \%$, and $61.1 \%$ of these anticipatory saccades $(\mathrm{SD}=25.7)$ went in the cued direction, thus further demonstrating participants difficulties in inhibiting the distractor gaze.

Figure 3 shows the saccade latencies for the remaining trials. An ANOVA with validity (cued vs. not cued), search target (pleasant target vs. threatening target) and emotion (fearful vs. happy) found a significant main effect of validity $\mathrm{F}(1,21)=31.8, p<0.0005$, whereby saccades were more rapidly executed in the cued than in any other

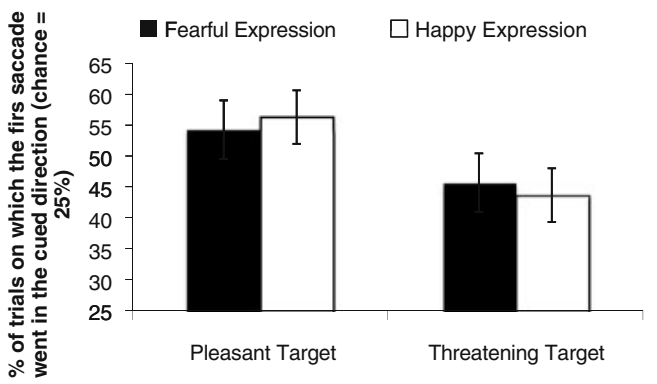

Fig. 2 The percentage of trials on which the first saccade went in the cued direction (i.e., gaze following), for fearful and happy expressions, in both the pleasant target as well as the threatening target conditions. The error bars represent the $95 \%$ confidence intervals. As there were four potential targets, random saccade directions would lead to $25 \%$ of trials on which the gaze went in the cued direction 
direction and thus demonstrating the expected cueing effect. There was a significant main effect of search target $\mathrm{F}(1,21)=6.12, p=0.022$, whereby saccades on pleasant target trials were initiated more rapidly than for threatening targets. No other main effects or interactions were significant (all $p$ values $>0.27$ ). While saccadic reaction times were significantly influenced by the gaze direction, this cueing effect was not affected by the emotional expression of the face, or the combination between the expression and the search target.

The final analysis looked at the way in which the distractor face influenced the target selection. In the pleasant target condition participants correctly identified the target on $80 \%(\mathrm{SD}=11.1)$ of trials, compared to $91 \%$ $(\mathrm{SD}=7.5)$ when searching for a threatening target. Classification performance on the threat search condition was significantly better than in the pleasant target condition $[\mathrm{t}(21)=6.8, p<0.0005]$. We defined the time to find the target as the point at which participants' pressed the space bar and thus indicating their selection. Figure 4 shows the time for selecting a target as a function of eye gaze and emotion for both search tasks. An ANOVA found a significant main effect of search task $\mathrm{F}(1,21)=13.5, p=$ 0.001 , indicating that peasant targets were found more rapidly than threatening ones. Moreover, there was a significant main effect of gaze $\mathrm{F}(1,21)=17.51, p<$ 0.0005 , demonstrating that targets that were looked at were detected more rapidly than those that were not looked at. However, there was no significant main effect of emotion $\mathrm{F}(1,21)=0.128, p=0.72$. The task by emotion interaction was also non significant $\mathrm{F}(1,21)=0.12, p=0.73$ and so was the emotion by eye gaze interaction $\mathrm{F}(1,21)=1.49$, $p=0.24$. Crucially the emotion by task by eye gaze interaction was non significant $\mathrm{F}(1,21)=1.35, p=0.26$. These results demonstrate that targets that were looked at by the face were detected more rapidly, but that this selection was independent of the emotional expression.

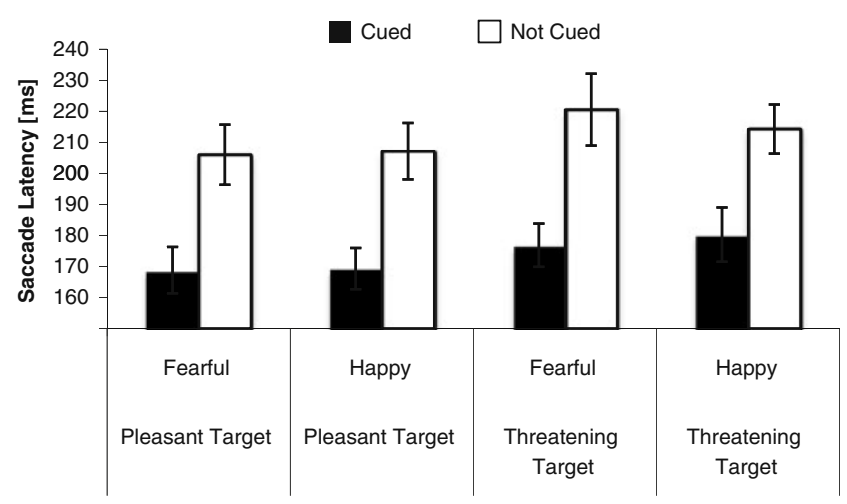

Fig. 3 Saccade latencies for the first saccade as a function of validity (cued vs. not cued) emotion (fearful vs. happy) and target (pleasant vs. threatening). Error bars denote standard errors

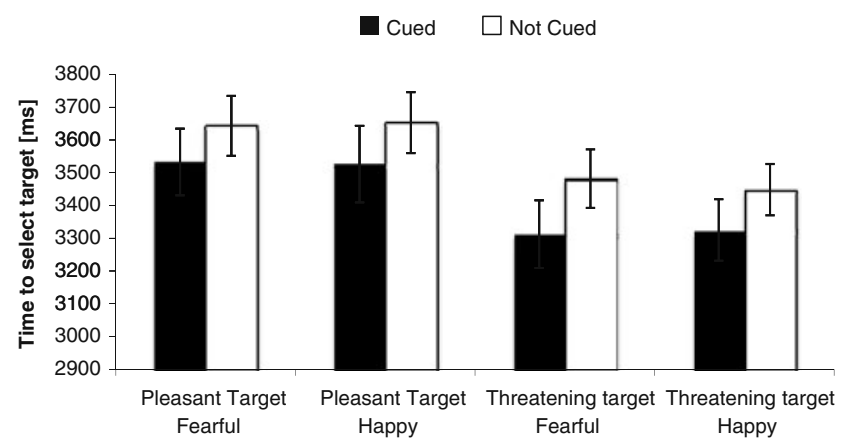

Fig. 4 Time taken for participants to select their target (space bar press) for targets that appeared in the cued locations (i.e., looked at by face) and not cued (i.e., not looked at face) as a function of emotion (fearful vs. happy) and target (pleasant vs. threatening). Error bars denote standard errors

\section{Discussion}

We used a visual search task to investigate the extent to which participants follow the gaze of a non-predictive and task irrelevant distractor gaze, and the way in which this gaze following was influenced by the emotional expression as well as participants' search task. Our results clearly illustrate a very strong tendency to initiate saccades in the direction in which a distractor gaze is looking, thus concurring with much of the past research (Kuhn \& Benson, 2007; Kuhn, et al., 2010; Kuhn \& Kingstone, 2009; Mansfield, et al., 2003; Ricciardelli, et al., 2002). As predicted, the degree to which the emotional expression influenced this gaze following varied as a function of the search goal. When searching for a threatening target, participants were more likely to look in the direction to which a fearful face was looking than a happy face. However, when searching for a pleasant target, this advantage for fearful faces disappeared. In fact in this condition, participants were numerically more inclined to follow the gaze of a happy than a fearful face, but this difference failed to reach statistical significance. Moreover, the non-significant main effect of emotion clearly demonstrates that fearful faces do not necessarily result in stronger gaze following than happy faces. The magnitude of the gaze following was not simply influenced by the emotional expression alone, but as a combination of the search goal and the emotional expression. The nonsignificant main effect of emotion demonstrates that fearful faces do not result in overall stronger gaze following.

In terms of saccade latencies, there was a strong cueing effect whereby participants initiated saccades more rapidly when the intended saccade direction was congruent to the distractor eyes. However, rather surprisingly, this cueing effect was independent of the emotional expression and the search goal. Similarly, cued targets were identified more rapidly than those that were not cued, but the target 
selection was not influenced by the emotional expression. Our results suggest an intriguing dissociation in the way that emotional expression influences attentional orienting in response to eye gaze. While the emotional expression and search target modulate the extent to which we look at locations being looked at by others, it seems to have no effect on how fast the saccade is initiated, or the time it takes for participants to find their target. Findlay and Walker (1999) proposed a model of saccade generation in which separate neurological pathways are responsible for programming the timing (i.e., when) and the directional (i.e., where) component of an eye movement. It is therefore possible that the way in which top down attentional control and the emotional expression influences eye movements is restricted to the where component of the saccade generation process. However, further research is required to confirm this conclusion. Rather intriguingly, participants initiated saccades more rapidly towards pleasant, than towards threatening targets. Similarly, pleasant targets were found more rapidly. These results suggest that people generally prefer orienting attention towards pleasant, rather than threatening stimuli, or may reflect subtle differences in visual saliency.

Most of the previous research on gaze cueing has employed rather simple Posner-type cuing tasks in which participants are required to detect a neutral search target (letters, or asterisk). More recently, Pecchinenda, Pes, Ferlazzo, and Zoccolotti (2008) have shown that participant's search task may indeed influence whether gaze cueing is affected by expression. Using a covert attention task, these authors demonstrated that when performing a neutral task (i.e., judging whether a word is written in upper or lower case), gaze cueing was independent of expression. However, when participants were required to perform an evaluative task (i.e., affective judgments about words), stronger cueing effects were found for negative (fearful and angry) than positive or neutral expressions. Participants in the current study performed an evaluative task in that they were asked to find the most pleasant or threatening animal. Pecchinenda et al. would predict stronger cueing effects for fearful than for happy expressions, independent of the search task. Whilst our methodology (covert vs. overt attention) as well as the exact nature of the task (visual search task vs. evaluative task) were rather different our results demonstrate that negative emotional expressions do not necessarily result in stronger cueing effects. However, it should be noted that as our task differed in several respects, any direct comparisons must be treated with caution, and future work will be required to explore these effects using a covert attentional task.

It has previously been suggested that gaze cueing of covert attention (Driver, et al., 1999; Friesen \& Kingstone, 1998) and gaze following is automatic and thus independent of top down control (Kuhn \& Kingstone, 2009). In the current experiment, the gaze cues were entirely task irrelevant and non-predictive of the target locations. Yet participants were three times more likely to initiate saccades in the cued rather than any of the other directions. This result demonstrates that we have great difficulties in inhibiting where another person is looking. Does this mean that gaze following is truly automatic? The fact that gaze following is modulated by emotional expression clearly illustrates that it is influenced by contextual factors. In the current study we go further in demonstrating that these contextual effects are themselves modulated by people's search task and thus under top down control. Recently, the evidence that was originally thought to reflect the effects of controlled, endogenous processing has been re-examined. For example, cues such as arrows have traditionally been thought to only enroll endogenous, controlled processes, yet their attentional effects resemble automatic attentional orienting to exogenous cues (Hommel, Pratt, Colzato, \& Godijn, 2001; Kuhn \& Benson, 2007; Kuhn, et al., 2010; Kuhn \& Kingstone, 2009; Tipples, 2002). Alternatively, the propensity by which exogenous cues capture attention is influenced by top down control (Folk, et al., 1992). Similarly, in terms of gaze following, whilst participants fail to inhibit gaze cues that are counter predictive of the intended saccade direction, the cueing effects found for these counter predictive cues were smaller than for nonpredictive cues, thus illustrating that the magnitude of gaze cueing is somewhat under top down control (Kuhn \& Kingstone, 2009). This rigid distinction between exogenous and endogenous attentional processes may be less fruitful since it is likely that few attentional processes are process pure (Ristic \& Kingstone, 2009). It may therefore be more useful to focus on what contextual and top-down factors affect gaze following and the way in which they interact with one another.

\section{References}

Driver, J., Davies, M., Kidd, P., Maxwell, M., Ricciardelli, P., \& Baron-cohen, S. (1999). Gaze perception triggers reflective visiospatial orienting. Visual Cognition, 6(5), 509-540.

Ekman, P., \& Oster, H. (1979). Facial expression of emotion. Annual Review of Psychology, 20, 527-554.

Fichtenholtz, H. M., Hopfinger, J. B., Graham, R., Detwiler, J. M., \& Labar, K. S. (2007). Happy and fearful emotion in cues and targets modulate event-related potential indices of gaze-directed attentional orienting. Social Cognitive and Affective Neuroscience, 2(4), 323-333.

Findlay, J. M., \& Walker, R. (1999). A model of saccade generation based on parallel processing and competitive inhibition. Behavioural and Brain Science, 22(4), 661-674. discussion 674-721.

Folk, C. L., Remington, R. W., \& Johnston, J. C. (1992). Involuntary covert orienting is contingent on attentional control settings. Journal of Experimental Psychology: Human Perception and Performance, 18(4), 1030-1044. 
Friesen, C. K., \& Kingstone, A. (1998). The eyes have it! Reflexive orienting is triggered by nonpredictive gaze. Psychonomic Bulletin \& Review, 5(3), 490-495.

Friesen, C. K., Ristic, J., \& Kingstone, A. (2004). Attentional effects of counterpredictive gaze and arrow cues. Journal of Experimental Psychology: Human Perception and Performance, 30(2), 319-329.

Frischen, A., Bayliss, A. P., \& Tipper, S. P. (2007). Gaze cueing of attention: Visual attention, social cognition, and individual differences. Psychological Bulletin, 133(4), 694-724.

Hietanen, J. K., \& Leppanen, J. M. (2003). Does facial expression affect attention orienting by gaze direction cues? Journal of Experimental Psychology: Human Perception and Performance, 29(6), 1228-1243.

Hommel, B., Pratt, J., Colzato, L., \& Godijn, R. (2001). Symbolic control of visual attention. Psychological Science, 12(5), 360 365.

Irwin, D. E., Colcombe, A. M., Kramer, A. F., \& Hahn, S. (2000). Attentional and oculomotor capture by onset, luminance and color singletons. Vision Research, 40(10-12), 1443-1458.

Kuhn, G., \& Benson, V. (2007). The influence of eye-gaze and arrow pointing distractor cues on voluntary eye movements. Perception \& Psychophysics, 69(6), 966-971.

Kuhn, G., Benson, V., Fletcher-Watson, S., Kovshoff, H., McCormick, C. A., Kirkby, J., et al. (2010). Eye movements affirm: Automatic overt gaze and arrow cueing for typical adults and adults with autism spectrum disorder. Experimental Brain Research, 201(2), $155-165$.

Kuhn, G., \& Kingstone, A. (2009). Look away! Eyes and arrows engage oculomotor responses automatically. Attention Perception \& Psychophysics, 71(2), 314-327.

Kuhn, G., \& Land, M. F. (2006). There's more to magic than meets the eye. Current Biology, 16(22), R950-R951.
Kuhn, G., Tatler, B. W., \& Cole, G. G. (2009). You look where I look! Effect of gaze cues on overt and covert attention in misdirection. Visual Cognition, 17(6), 925-944.

Langton, S. R. H., Watt, R. J., \& Bruce, V. (2000). Do the eyes have it? Cues to the direction of social attention. Trends in Cognitive Sciences, 4(2), 50-59.

Mansfield, E. M., Farroni, T., \& Johnson, M. H. (2003). Does gaze perception facilitate overt orienting? Visual Cognition, 10(1), 7 14

Mathews, A., Fox, E., Yiend, J., \& Calder, A. (2003). The face of fear: Effects of eye gaze and emotion on visual attention. Visual Cognition, 10(7), 823-835.

Pecchinenda, A., Pes, M., Ferlazzo, F., \& Zoccolotti, P. (2008). The combined effect of gaze direction and facial expression on cueing spatial attention. Emotion, 8(5), 628-634.

Putman, P., Hermans, E., \& Van Honk, J. (2006). Anxiety meets fear in perception of dynamic expressive gaze. Emotion, 6(1), 94102.

Ricciardelli, P., Bricolo, E., Aglioti, S. M., \& Chelazzi, L. (2002). My eyes want to look where your eyes are looking: exploring the tendency to imitate another individual's gaze. NeuroReport, 13 (17), 2259-2264.

Ristic, J., \& Kingstone, A. (2009). Rethinking attentional development: Reflexive and volitional orienting in children and adults. Developmental Science, 12(2), 289-296.

Tipples, J. (2002). Eye gaze is not unique: Automatic orienting in response to uninformative arrows. Psychonomic Bulletin \& Review, 9(2), 314-318.

Tipples, J. (2006). Fear and fearfulness potentiate automatic orienting to eye gaze. Cognition \& Emotion, 20(2), 309-320.

Vecera, S. P., \& Rizzo, M. (2006). Eye gaze does not produce reflexive shifts of attention: Evidence from frontal-lobe damage. Neuropsychologia, 44(1), 150-159. 